\section{Vasectomy: is the apparent decline real or not?}

\section{Nahid Punjani and Marc Goldstein}

Trends in vasectomy use are controversial, but rates might be declining over time. Several factors could contribute to this apparent decrease, which warrant consideration before definitive conclusions are made.

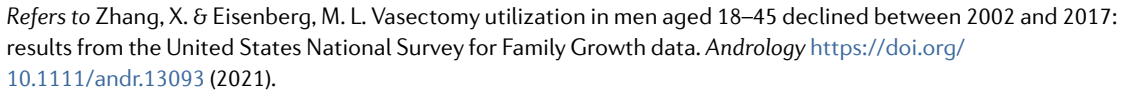

Vasectomy, a simple procedure for permanent sterilization that involves division of the vas deferens, dates back to the $1800 \mathrm{~s}^{1}$. The fundamental tenets of this procedure have remained unchanged, but technical advances include implementation of the no-scalpel vasectomy ${ }^{2}$. Owing to its relative simplicity, high success rates and very low complication rates, vasectomy is the most common method of permanent contraception in men, which makes sense given the limited alternative options for male contraception ${ }^{3,4}$. Furthermore, in spite of vasectomy being far more effective and safer, tubal ligation is still performed far more often worldwide than vasectomy.

Controversy regarding trends in vasectomy use is ongoing, which is especially interesting given a clear trend to delay starting a family for both men and women ${ }^{5}$. In the recent study by Zhang and Eisenberg ${ }^{6}$, they cleverly harnessed information from available public data to answer the simple question of what the trends in vasectomy use are ${ }^{6}$. Using a robust dataset, the National Survey for Family Growth, which provides national representation in the USA, the authors report that rates of vasectomy were decreasing between 2002 and 2017 (REF. ${ }^{7}$ ). These findings persisted across multiple age groups and after adjustment for potential confounding factors. They also demonstrated multiple factors associated with vasectomy, including age, race, marital status, education, birth outside the USA, income, religion and number of biological children. These important findings warrant further consideration and interrogation.

A well-known limitation of these findings is that the exclusive focus was on the USA, which, while meaningful, does limit its generalizability to other countries. This limitation applies especially and importantly to those countries with a national health-care system, such as Canada or the UK. Access issues are probably different from the USA in these regions, for example, wait times and litigious implications might be different. That said, data in these regions pertaining specifically to vasectomy patterns remain sparse and require further study; seeing how they correlate with data from the USA would be interesting.

Some interesting data missing from this study, which might provide further insight into its validity, include partner data, such as their age (as we know this factor is the most important factor predicting fertility), whether they had or considered a sterilization procedure and/or whether they were using any form of contraception. Some data have suggested declines in the rate of female sterilization, but it would be worthwhile to see whether recent national data complement or oppose the decrease observed in vasectomy over time ${ }^{8}$. The declines in female sterilization have been suggested to be a result of the numerous contraceptive options available to women that are effective, not permanent and less invasive.

One of the most interesting findings is the change in the absolute proportion of individuals seeking vasectomy who have zero biological children ${ }^{6}$. In 2002, this rate was $7.5 \%$, the lowest rate was reported from 2006 to 2010 at $3.3 \%$ but peaked at $12.4 \%$ between 2015 and 2017. Perhaps these individuals are either not interested in having children or they are
Controversy regarding trends in vasectomy use is ongoing

expanding their family through other means, such as adoption. Investigation of this finding is warranted and study in a prospective setting would be informative.

Additionally, changes in laws and acceptance of same sex marriage, gender reassignment and abortion might contribute to the findings of this study ${ }^{6}$. With respect to same sex marriage and/or gender reassignment, a decrease in vasectomy might be apparent for couples who are unable to conceive naturally, who are required, depending on their circumstances, to use surrogacy, donor sperm, donor oocytes or adoption. Again, data on the partner of these men in the dataset would be important to further understand the trends. On the other hand, changes in abortion laws might also be an important indicator of the trend seen for rates of vasectomy. In regions where abortion is acceptable and permitted, rates of vasectomy might be lower, but could be higher where abortion is not allowed; this information is not available in the report by Zhang and Eisenberg ${ }^{6}$.

Moving forward, given previous reports demonstrating an increase in vasectomy use during the Great Recession, observing the impact of the COVID-19 pandemic on vasectomy rates will be interesting 9 . COVID-19 has created economic strain, modified many family and couple dynamics, altered many living situations and especially increased the number of individuals working from home. These modified environments probably affect family planning decisions. Anecdotally, in clinical practice, we have witnessed a decrease in vasectomies but an increase in vasectomy reversal, suggesting an interest in family expansion.

Given the permanent nature of vasectomy, the delay in family planning and the advances in assisted reproductive technology, whether the trend away from vasectomy is true remains debatable. In some ways, the results of this study ${ }^{6}$ contradict the recent report of a global decline in birth rates before and after the pandemic, which reinforces the importance of this question and interrogation of these data ${ }^{10}$. That said, alternative 


\section{4 observing the impact of the COVID-19 pandemic on vasectomy rates will be interesting}

non-permanent forms of male contraception are limited, but non-permanent female options are plentiful, effective and are a crucial part of the discussion.

In summary, these findings warrant further study and consideration of possible confounding factors. Global work on a larger scale is needed to determine the meaning of these trends, especially as global birth rates decline.
Nahid Punjani and Marc Goldstein ${ }^{凶}$ Center for Reproductive Medicine and Surgery, Institute for Reproductive Medicine and Department of Urology, Weill Cornell Medicine, New York, $N Y, U S A$.

凶e-mail:mgoldst@med.cornell.edu

\section{https://doi.org/10.1038/s41585-021-00538-1}

1. Sheynkin, Y. R. History of vasectomy. Urol. Clin. North Am. 36, 285-294 (2009).

2. Li, S. Q., Goldstein, M., Zhu, J. \& Huber, D. The no-scalpel vasectomy. J. Urol. 145, 341-344 (1991).

3. Haldar, N., Cranston, D., Turner, E., MacKenzie, I.

$\&$ Guillebaud, J. How reliable is a vasectomy?

Long-term follow-up of vasectomised men. Lancet 356, 43-44 (2000).

4. Kavanaugh, M. L. \& Jerman, J. Contraceptive method use in the United States: trends and characteristics between 2008, 2012 and 2014. Contraception 97 14-21 (2018).

5. Balasch, J. \& Gratacós, E. Delayed childbearing effects on fertility and the outcome of pregnancy. Fetal Diagn. Ther. 29, 263-273 (2011).
6. Zhang, X. \& Eisenberg, M. L. Vasectomy utilization in men aged 18-45 declined between 2002 and 2017 : results from the United States National Survey for Family Growth data. Andrology https://doi.org/ 10.1111/andr. 13093 (2021).

7. Eisenberg, M. L. \& Lipshultz, L. I. Estimating the number of vasectomies performed annually in the United States: data from the National Survey of Family Growth. J. Urol. 184, 2068-2072 (2010).

8. Chan, L. M. \& Westhoff, C. L. Tubal sterilization trends in the United States. Fertil. Steril. 94, 1-6 (2010).

9. Sharma, V. et al. Relating economic conditions to vasectomy and vasectomy reversal frequencies: a multi-institutional study. J. Urol. 191, 1835-1840 (2014).

10. Bricker, D. Bye, bye, baby? Birthrates are declining globally-here's why it matters. World Economic Forum https://www.weforum.org/agenda/2021/06/ birthrates-declining-global-fertility-decline-emptyplanet-covid-19-urbanization-migration-population/ (2021).

\section{Competing interests}

The authors declare no competing interests. 